\title{
Flow-induced vibrations of offshore flare towers and flare booms
}

\author{
G. Moe ${ }^{1} \&$ J. M. Niedzwecki ${ }^{2}$ \\ ${ }^{I}$ Department of Civil and transport Engineering, NTNU, Trondheim, \\ Norway \\ ${ }^{2}$ Department of Civil Engineering, Texas A\&M University, \\ College Station, TX, USA
}

\begin{abstract}
A historical perspective on the performance of vertical and inclined flare structures is provided to illustrate the breadth of the problem and provide a basis to appreciate the engineering solutions to the issue of flow-induced vibrations.

Keywords: flare booms, flare towers, flow-induced vibrations, wind-induced vibrations, vortex-induced vibrations, fatigue damages, fatigue cracks.
\end{abstract}

\section{Introduction}

On offshore platforms used for the production of oil and/or gas there is a special structure whose function is to lead any excess natural gas away from the platform to a burner located sufficiently far away from the platform. These structures are denoted flare towers if their main axis is nearly vertical, or flare booms if their longitudinal axis is inclined. In the North Sea these are truss structures of a length on the order of $100 \mathrm{~m}$. A large number of cracks have been found in several of these flare structures, but it is striking that even though many cracks have been discovered and even though they sometimes are more than 20 year old, none the cracks have grown large enough to fracture through an entire cross section. One plausible hypothesis is that the structural damping increases considerably when cracks have been developed.

\section{A review of some historic events}

From the Norwegian sector of the North Sea there were reports on at least 8 flare booms or flare towers that experienced damage from vibrations believed to be of 
the flow-induced type. According to Kvitrud [6] 45 cracks were detected in the flare booms of Statfjord B and Statfjord C. In 1990 Kvitrud and Karlsen [7] reported that 19 cracks were found on Statfjord B and most of them were rather long. Three of the cracks were analyzed in detail and it was found that they were oxidized all the way to the root, indicating that they had existed for a long time but had not grown recently and no fresh cracks were found. On Gullfaks $B$ in 1992 nine cracks were found in the flare structure. In 198710 cracks were reported in the flare tower on Odin, and in 198910 cracks were reported in the Vallhall flare boom, see Oppen and Kvitrud [11] for more details. In the British sector, an OTC paper by Bell and Morgan [1] reports on 10 cracks in the Murchison flare boom. Similarly in the flare tower on the Danish Gorm D platform, seven cracks were reported.

Because of quite noticeable vibrations on the Statfjord A and Heimdal platforms, remedial action was taken at an early stage. On the Statfjord $A$ platform the corrective actions took place in 1978, and consisted of reparation of cracks and installation of vortex suppression devices in the form of spiral wrapping of $25 \mathrm{~mm}$ ropes on the main chords. According to oral information this structure was inspected again in 1989 and only very modest damage was detected. The Heimdal flare boom was installed in August 1985 and fairly dramatic vibrations were noticed in November and December of the same year. The noise and the shaking of floors triggered immediate attention from platform residents who wasted no time in notifying the leadership of the situation. Subsequently, spiral wrapping of ropes on some structural members was implemented as a temporary fix. Three consultant companies were engaged and presented their report on this matter in April 1985, and this was immediately followed by wind tunnel model tests to better understand the problem and possible engineering solutions. In the summer of 1986, vortex suppression devices were installed in the form of shrouds on several members and post tensioned ties between certain members. As a reaction to the Heimdal events, alternative design flare tower philosophies were adopted. The Oseberg A platform was designed so that all flare structural members had critical wind velocities higher than the design wind velocity, i.e. the frequencies at which VIV would occur were avoided by stiffening all parts of the structure. According to Sjursen [13] this lead to a unit steel weight that was almost three times as large as for the Heimdal flare boom. Subsequently it was attempted to find safe, but not overly conservative design approaches, as shown later in this paper.

\section{Design rules}

Structural damping is presented here in terms of the damping ratio $\zeta$, i.e. the ratio of the damping force to its critical value, and to obtain the logarithmic decrement, $\zeta$ should be multiplied by $2 \pi$. In practice the determination of the damping ratio is difficult. Still many researchers have obtained such estimates by various methods, and while large variability remains some degree of consensus has been reached on the value $\zeta=0.15 \%$. This was prescribed in the British Standard BS8100 as early as 1986, and today this value may still seem a 
sensible choice. Thus in NORSOK N-003 it is stated: "The material and structural damping of individual elements in welded steel structures should not be set larger than $0.15 \%$ of critical damping when vortex induced vibrations are considered", while in DNV-RP-C205 it is similarly stated: "For wind exposed steel members, the structural damping ratio $\left(\delta_{s} / 2 \pi\right)$ may be taken as 0.0015 , if no other information is available".

A classic approach for the design of flare booms in natural wind is to start from the modal amplitude for a single degree of freedom system consisting of a section model on springs in steady flow, and then determine the motion amplitude $y_{0}$ as a function of the combined stability parameter, (or 'Scruton number')

$$
K_{s}=4 \pi \frac{m}{\rho D^{2}} \zeta
$$

For a modal shape $y(x)$ with arbitrary amplitude, the shape parameter $\gamma$ determines the modal displacements as $\gamma y(x)$, in which

$$
\gamma=y_{0}\left\{\frac{\int_{0}^{L} y^{2}(x) d x}{\int_{0}^{L} y^{4}(x) d x}\right\}^{\frac{1}{2}}
$$

This approach is used in DNV-RP-C205. The theory behind the formula for $\gamma$ above was developed by Iwan [5] using a nonlinear dynamic model, a van der Pol oscillator. It is presented in Blevins [2] and but requires a number of nonobvious assumptions, see Moe [8], so it should probably only be viewed as an empirical formula.

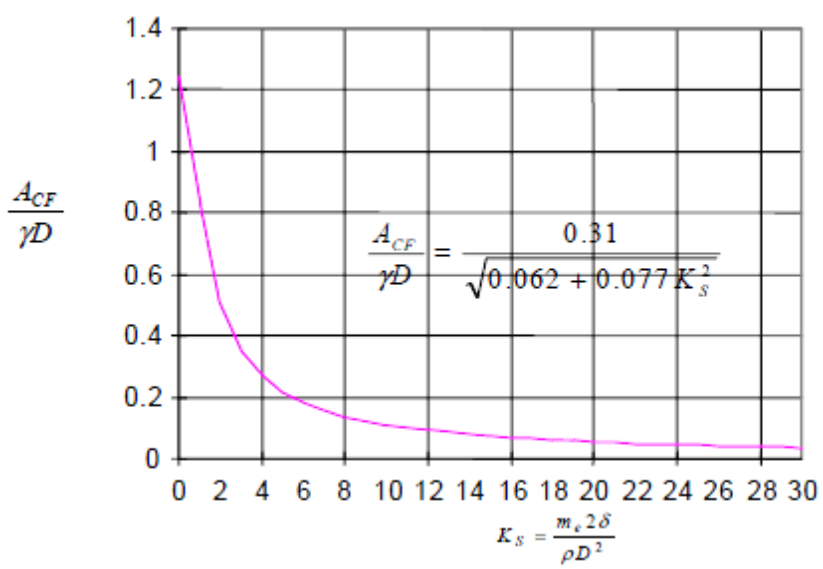

Figure 1: Cross flow motion amplitude according to DNV-RP-C205.

After the Heimdal incident alternative formulations to obtain more realism were attempted. One important consideration is the variability of the wind velocity in both space and time. For a harmonic excitation at resonance and a displacement that is starting from zero elementary theory says that the vibration 
amplitude will be $[1-\exp (-\zeta \omega t)]$ times the steady state solution. Thus, for a damping ratio $\zeta=0.15$ it will take about 74 oscillation periods to reach just $50 \%$ of the steady state amplitude, and about 245 oscillations to reach $90 \%$ of the steady state amplitude. This applies to a perfectly synchronized harmonic loading. However VIV forcing is much less organized especially initially when the amplitudes are small, so one must expect that several hundred oscillations will be required to reach large VIV amplitudes. As will be seen later in the Heimdal case one had larger motions than predicted by the above theory, not as is argued here, smaller vibrations, thus some other explanations are called for. A good presentation of some of the alternative VIV prediction models is given in Rudge and Fei [12]. Recently a suite of Eurocodes has been developed, and these are now being augmented by national annex kits. Space does not permit any further discussion of this, but it may be mentioned that the procedures are quite complicated and that the modeshape factor (E.9) in Eurocode 1-4, which is most relevant for our purpose, differs substantially from $\gamma$, the modeshape factor quoted earlier in this section.

\section{Flow-induced vibrations}

The observed vibrations are clearly caused by the wind, and are usually referred to as 'vortex induced vibrations' (VIV) in the literature, but in the author's opinion that is too narrow a designation, and the term 'flow-induced vibrations' (FIV) should be preferred. There are many types of FIV, but disregarding situations in which vibrations are due to internal flow, the two most familiar FIV phenomena are probably VIV and galloping. VIV is driven by individual vortices, while for galloping the shedding is so fast compared to the structural vibrations that these are governed by averaged lift forces depending on the relative motions between fluid and structure, i.e. the change in the lift force versus inflow angle. Galloping may occur even for circular cross sections, due to e.g. marine growth or accumulation of ice or dirt. A third mechanism, Flow interaction among groups of cylinders, is very often a problem and may occur in a number of different constellations, such as tandem arrangements, 'side-by-side' arrangements, rectangular patterns, centralized patterns, cylinders with 'kill-andchoke lines, proximity to a wall or to a large, rigid object, etc. Turbulence induced vibrations, so-called buffeting will often also be classified as FIV, even though this case usually are dealt with by the classical random vibration theory. For flare booms buffeting is usually not critical. One justification for this statement can be had from the fact that the vibrations in question usually occur in the cross-flow direction.

\section{Wake-induced vibrations}

Wake-induced vibrations are a subgroup of flow interaction vibrations, and may occur in situations in which one or more cylinders are in the wake of an upstream cylinder, i.e. in tandem arrangements. The case of 2 equal diameter cylinders 
has been dealt with in a large number of papers and textbooks. A situation with one cylinder in the wake of a larger upstream cylinder may be even more interesting, since the wake from a larger upstream cylinder is stronger than that created by the vibrating cylinder itself. An experimental investigation of such a situation was presented by Moe et al. [9]. It was hypothesized that the diagonals on the Heimdal flare boom were vibrating due to wind parallel to one face of the boom so that the diagonals were in the wake of the leg members. Indeed the vibrations appeared to occur in wind precisely parallel to one face of the boom. In that case the diameter ratio was about 2.0 and in the wind tunnel experiments a simpler geometry and diameter ratios of 1.8 and 2.2 were used, see figure 2 .

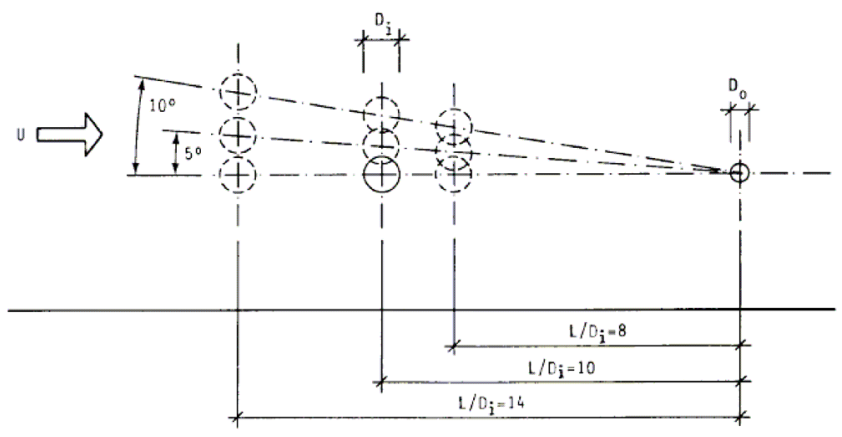

Figure 2: Side view of the rig for the tandem arrangement tests. Wind from the left, test cylinder on the downwind side. Upstream cylinder at various distances and angles, relative to the test cylinder, giving nine configurations in total. From Moe et al. [9].

The larger, front cylinder was fixed, the smaller, rear cylinder, 'the test cylinder', was spring-supported as shown in figure 3. It is seen that the test cylinder is at a relatively large distance from the front cylinder.

The vibration amplitudes are plotted in figure 4 and it is seen that the smaller cylinder vibrates at roughly the double amplitude when it is in the wake of the larger cylinder, as compared to the stand-alone case. Also maximum vibrations occur when the wake frequency is equal to the natural frequency of the vibrating cylinder $f_{n}$, i.e. at higher wind velocities than what creates VIV for the stand alone case. The reduced velocity at the test cylinder is by definition:

$$
U_{\text {red,test }}=\frac{U}{f_{n} D_{\text {test }}}
$$

The vortex shedding frequency of the front cylinder (and the wake) $f_{\text {front }}$ follows from

$$
U_{\text {red }, 0}=\frac{U}{f_{\text {front }} D_{\text {front }}} \text { or } \quad f_{\text {front }}=\frac{U}{U_{\text {red }, 0} D_{\text {front }}}
$$




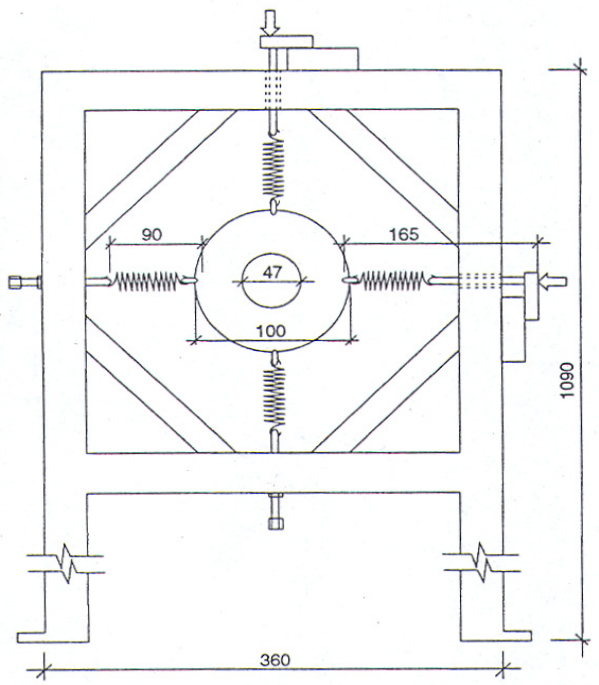

Figure 3: One of the two supporting frames for the test cylinder, seen from the side, i.e. this frame is in a vertical plane parallel to the airflow. The test cylinder is seen as the smallest circle in the figure; it is marked ' 47 '. Both frames are mounted on a $500 \mathrm{~kg}$ concrete block. Note that the length unit is millimeters.

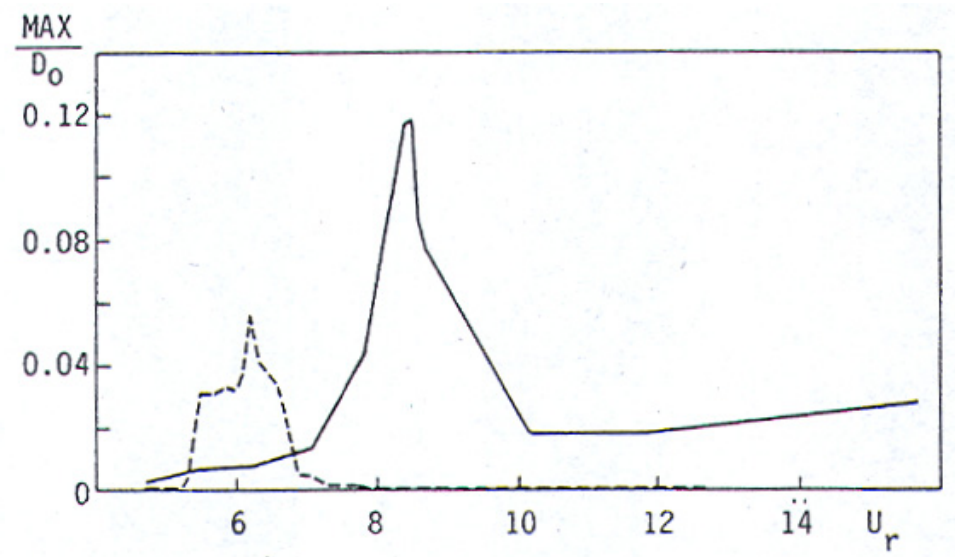

Figure 4: Plot of single cylinder vibration amplitudes (dashed line) and vibration amplitudes of the same cylinder in the wake of an $80 \%$ larger, fixed cylinder (solid line). Both are plotted versus reduced velocity of the test cylinder whose diameter is $D_{0}$, i.e. $U_{r}=U / f_{n} D_{0}$. Note that both cylinders are smooth and the Reynolds number is in the subcritical range. 
The reduced velocity $U_{\text {red, } 0}$ is in this case approximately 5 , since the flow is in the subcritical range and the front cylinder is fixed. Then setting

$$
f_{n}=f_{\text {front }} \Rightarrow U_{\text {red, test }}=\frac{U}{f_{\text {front }} D_{\text {test }}}=\left(\frac{U_{\text {red }, 0} D_{\text {front }}}{U}\right) \frac{U}{D_{\text {test }}}=U_{\text {red }, 0} \frac{D_{\text {front }}}{D_{\text {test }}}
$$

Thus maximum VIV motions are expected at about $5 \times 1.8=9$, which is in excellent agreement with the experimental results shown in figure 4.
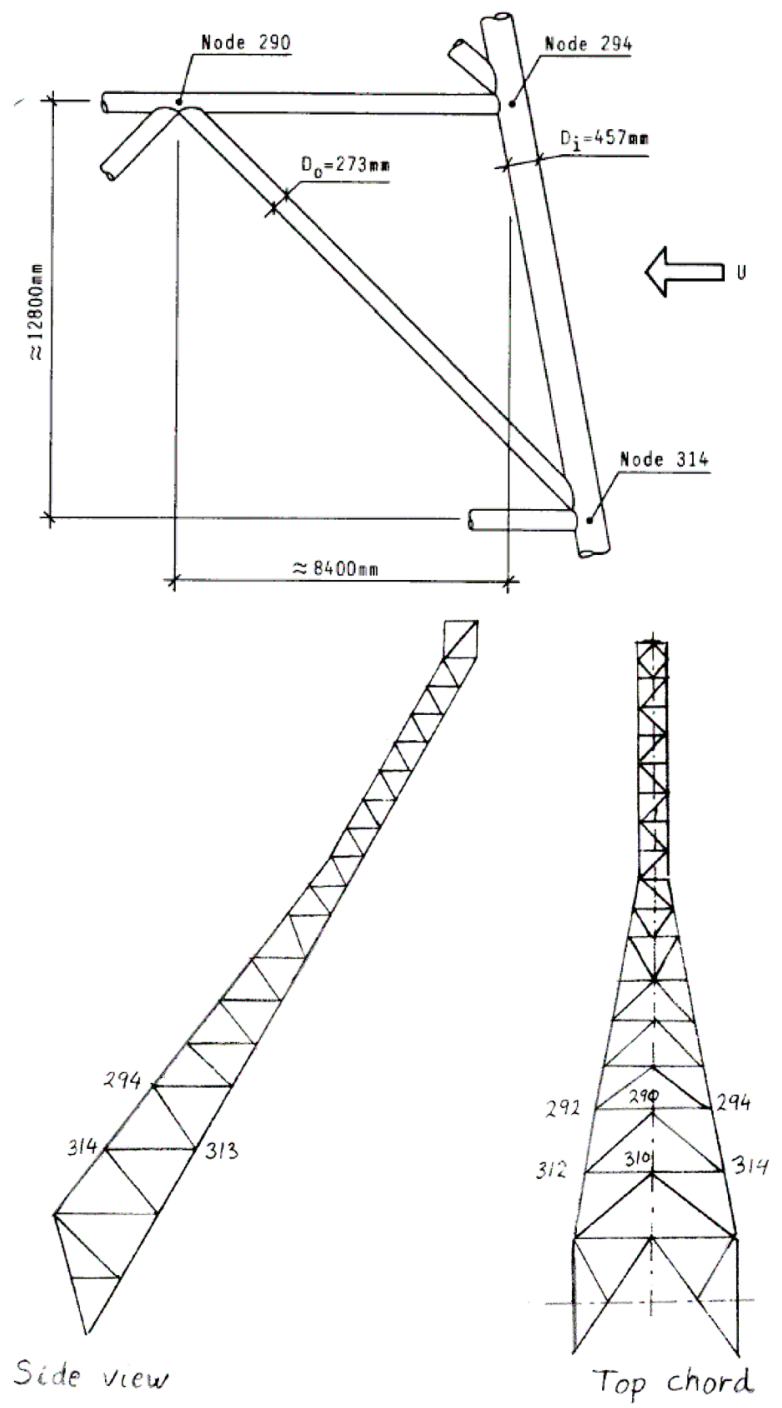

Figure 5: The Heimdal flare boom. 


\section{The Heimdal flare boom}

The report on the initial Heimdal incidents referred to vibrations in the areas where the structural members are numbered in figure 5, and these were most often designated as "vibrating as a frame." Typical amplitudes were "min 50 mm" for a frequency of $3 \mathrm{~Hz}$. In Moe 1989 it was found $K_{s}=18.2$ based on $\zeta=0.2$, thus if instead $\zeta=0.15$ is used one would get $K_{s}=13.7$, which according to the DNV curve in Figure 1would yields the prediction $A_{\mathrm{y}} /(\gamma D)=0.08$. Using a lower limit of the observed amplitude, $50 \mathrm{~mm}$, a shape factor of 1.155 and a diameter $D=173 \mathrm{~mm}$. this results in an observed ratio of $A_{\mathrm{y}} /(\gamma D)=0.16$. Thus the observed vibration amplitude is at least the double of the predicted value. Further, the vibrations occurred at wind velocities that did not match the reduced velocity at VIV. These large vibrations occurred only for very special wind directions, namely parallel to the top and bottom faces of the boom. Unfortunately these conclusions are not absolutely certain, since misrepresentation or misinterpretation of the data cannot be ruled out. For example there remain questions such as: 1.) Has the wind velocity been given at the local elevation or has the velocity at $10 \mathrm{~m}$ been backfigured? 2.) Are the calculated local frequencies correct?

\section{Concluding remarks}

Rather significant cracking has been observed on several flare booms (or flare towers) in the Norwegian sector, and some in other geographical areas. One attempt to design flare booms so that flow induced vibrations could not occur lead to a very significant weight increase. Various vortex suppression devices have been successful in the two cases for which documentation is available, and consisted of a temporary solution using helical wound ropes followed by a permanent solution with shrouds and post-tensioned ties on Heimdal, and helically wound ropes on Statfjord A.

\section{References}

[1] Bell, E. R. G., and Morgan, D .G., "Repair and analysis of cracking in the Murchison flare boom”, OTC 5814, Offshore Technology Conference, Houston, Texas, 1988

[2] Blevins, R.D. Flow-induced Vibrations, 2nd ed, 1990, van Nostrand Reinhold, New York

[3] DNV-RP-C205, Environmental Conditions and Environmental Loads,

[4] Eurocode 1-4, Eurocode 1 Actions on structures - Part 1-4 General actions - Wind Actions, EN 1991-1-4, April 2005

[5] Iwan, W.D., (1975) "The vortex induced oscillation of elastic structural elements", J of Engineering for Industry, 97, 1378-1382

[6] Kvitrud, A. 1994, http://home.c2i.net/kvitrud/Arne/Wind-inducedproblems-in-Norway.htm 
[7] Kvitrud, A. Karlsen, K., "Resonant transverse vibrations in wind (In Norwegian)", Report OD_93-77, The Norwegian Petroleum Directorate, Stavanger, Norway, September 1993

[8] Moe, G., An experimentally based model for prediction of lock-in riser motions, Proceedings of OMAE-91, Vol 1-B, p497-506, 1991

[9] Moe G, Domben T, Steen PE: "Vibration of a Cylinder in the wake of a Larger Cylinder", Proceedings Second European Conference on Structural Dynamics, Eurodyn '93, AA Balkema, Rotterdam, pp. 1083-1090, 1993

[10] NORSOK Standard N-003, Ed. 2, "Action and action effects", Sept. 2007

[11] Oppen, A N, Kvitrud, A, "Wind induced resonant cross flow vibrations on Norwegian offshore flare booms", OMAE 1995, Volume 1-B, pp. 341-354

[12] Rudge, D., Fei, C.-Y., "Response of Structural Members to Wind-Induced Vortex Shedding", MSC dissertation, MIT, 1988

[13] Sjursen, K., Avoidance Criteria for Wind-Induced Vortex Shedding Vibrations, Proceedings of ISOPE 1999 